\title{
The Global Attractors for the Higher-Order Kirchhoff-Type Equation with Nonlinear Strongly Damped Term
}

\author{
Yuting Sun, Yunlong Gao, Guoguang Lin \\ Mathematical of Yunnan University, Kunming, China \\ Email: syt19911006@163.com,gyl0813101x@163.com,gglin@ynu.edu.cn
}

How to cite this paper: Sun, Y.T., Gao, Y.L. and Lin, G.G. (2016) The Global Attractors for the Higher-Order KirchhoffType Equation with Nonlinear Strongly Damped Term. International Journal of Modern Nonlinear Theory and Application, 5, 203-217.

http://dx.doi.org/10.4236/ijmnta.2016.54019

Received: October 27, 2016

Accepted: November 25, 2016

Published: November 28, 2016

Copyright ( $) 2016$ by authors and Scientific Research Publishing Inc. This work is licensed under the Creative Commons Attribution International License (CC BY 4.0).

http://creativecommons.org/licenses/by/4.0/

\section{(c) (i) Open Access}

\section{Abstract}

We investigate the global well-posedness and the global attractors of the solutions for the Higher-order Kirchhoff-type wave equation with nonlinear strongly damping: $u_{t t}+\sigma\left(\left\|\nabla^{m} u\right\|^{2}\right)(-\Delta)^{m} u_{t}+\phi\left(\left\|\nabla^{m} u\right\|^{2}\right)(-\Delta)^{m} u=f(x)$. For strong nonlinear damping $\sigma$ and $\phi$, we make assumptions $\left(\mathrm{H}_{1}\right)-\left(\mathrm{H}_{4}\right)$. Under of the proper assumption, the main results are existence and uniqueness of the solution in $H^{2 m}(\Omega) \times H_{0}^{m}(\Omega)$ are proved by Galerkin method, and deal with the global attractors.

\section{Keywords}

Strongly Nonlinear Damped, Higher-Order Kirchhoff Equation, The Existence and Uniqueness, The Global Attractors

\section{Introduction}

We consider the following Higher-order Kirchhoff-type equation:

$$
\begin{gathered}
u_{t t}+\sigma\left(\left\|\nabla^{m} u\right\|^{2}\right)(-\Delta)^{m} u_{t}+\phi\left(\left\|\nabla^{m} u\right\|^{2}\right)(-\Delta)^{m} u=f(x),(x, t) \in \Omega \times[0,+\infty), \\
u(x, t)=0, \frac{\partial^{i} u}{\partial v^{i}}=0, i=1,2, \cdots, m-1, x \in \partial \Omega, t \in(0,+\infty), \\
u(x, 0)=u_{0}(x), u_{t}(x, 0)=u_{1}(x), x \in \Omega,
\end{gathered}
$$

where $m>1$ is an integer constant, and $\Omega$ is a bounded domain of $R^{n}$, with a smooth dirichlet boundary $\partial \Omega$ and initial value. Moreover, $v$ is the unit outward normal on $\partial \Omega . \sigma$ and $\phi$ are scalar functions specified later, $f$ is a given function. 
This kind of wave models goes back to G. Kirchhoff [1] and has been studied by many authors under different types of hypotheses. There have been many researchers on the global attractors existence of Kirchhoff equation, we can refer [2] [3] [4] [5] [6]. What's more, the global attractors for the Higher-order Kirchhoff-type equation are investigated and we refer to [7] [8] [9].

Zhijian Yang and Pengyan Ding [2] studied the longtime dynamics of the Kirchhoff equation with strong damping and critical nonlinearity on $R^{n}$ :

$$
u_{t t}-\Delta u_{t}-M\left(\|\nabla u\|^{2}\right) \Delta u+u_{t}+g(x, u)=f(x) .
$$

They establish the well-posedness, the existence of the global and exponential attractors in natural energy space $H=H^{1}\left(R^{N}\right) \times L^{2}\left(R^{N}\right)$ in critical nonlinearity case. On this basis, they also investigated the global well-posedness and the longtime dynamics of the Kirchhoff equation with fractional damping and supertical nonlinearity [3]:

$$
u_{t t}-M\left(\|\nabla u\|^{2}\right) \Delta u+(-\Delta)^{\alpha} u_{t}+f(u)=g(x), \quad \text { with } \alpha \in\left(\frac{1}{2}, 1\right) .
$$

The main results are focused on the relationships among the growth exponent $p$ of the nonlinearity $f(u)$, the global well-posedness and the longtime dynamics of the equations. They show that i) even if $p$ is up to the supercritical range, that is,

$1 \leq p \leq \frac{N+4 \alpha}{(N-4 \alpha)^{+}}$, the well-posedness and the longtime behavior of the solutions of the equation are the characters of the parabolic equation; ii) when

$\frac{N+4 \alpha}{(N-4 \alpha)^{+}} \leq p<\frac{N+4}{(N-4)^{+}}$, the corresponding subclass $G$ of the limit solutions exists and possesses a weak global attractors.

Varga Kalantarov and Sergey Zelik [5] present a new method of investigating the so-called quasi-linear strongly damped wave equations:

$$
\partial_{t}^{2} u-\gamma \partial_{t} \Delta_{x} u-\Delta_{x} u+f(u)=\nabla_{x} \cdot \phi^{\prime}\left(\nabla_{x} u\right)+g .
$$

In bounded 3D domains. This method establishes the existence and uniqueness of energy solutions in the case where the growth exponent of the non-linearity $\phi$ is less than 6 and $f$ may have arbitrary polynomial growth rate. Moreover, the existence of a finite-dimensional global and exponential attractors for the solution semigroup associated with that equation and their additional regularity are also established. In a particular case $\phi \equiv 0$ which corresponds to the so-called semi-linear strongly damped wave equation, their result allows to remove the long-standing growth restriction $|f(u)| \leq C\left(1+|u|^{5}\right)$. The Cauchy problem and the boundary value problem for equation under the different assumptions on the nonlinearities $\phi$ and $f$ have been studied in many papers, but the author uses a new method to this equation.

Xiuli Lin and Fushan Li [6] consider the initial-boundary value problem for nonlinear Kirchhoff-type equation: 


$$
\begin{array}{ll}
u_{t t}-\varphi\left(\|\nabla u\|_{2}^{2}\right) \Delta u-a \Delta u_{t}=b|u|^{\beta-2} u, & \text { in } \Omega \in(0, \infty), \\
u(x, t)=0, & \text { on } \Gamma_{1} \times(0, \infty), \\
\varphi\left(\|\nabla u\|_{2}^{2}\right) \frac{\partial u}{\partial v}+a \frac{\partial u_{t}}{\partial v}=g\left(u_{t}\right), & \text { on } \Gamma_{0} \times(0, \infty), \\
u(u)=u_{0}, u_{t}(x, 0)=u_{1}, & \text { in } \Omega .
\end{array}
$$

where $a, b>0$ and $\beta>2$ are constants, $\varphi$ is a $C^{1}$-function such that $\varphi(s) \geq \lambda_{0}>0$ for all $s \geq 0$. Under suitable conditions on the initial data, they show the existence and uniqueness of global solution by means of the Galerkin method and the uniform decay rate of the energy by an integral inequality. Here, $\varphi(s)$ satisfying $\varphi(s) \geq m_{0}>1$ and $s \varphi(s) \geq \int_{0}^{s} \varphi(\tau) \mathrm{d} \tau, \forall s \in(0, \infty)$. In this paper, for strong nonlinear damping $\sigma$ and $\phi$, we make some similar assumptions. These assumptions will be presented in the following statements.

In 2004, Fucai Li [7] dealed with the higher-order Kirchhoff-type equation with nonlinear dissipation:

$$
u_{t t}+\left(\int_{\Omega}\left|D^{m} u\right|^{2} \mathrm{~d} x\right)^{q}(-\Delta)^{m} u+u_{t}\left|u_{t}\right|^{r}=|u|^{p} u, x \in \Omega, t>0
$$

In a bounded domain, where $m>1$ is a positive integer, and $q, p, r>0$ are positive constants. They obtain that the solution exists global if $p \leq r$, while if $p>\max \{r, 2 q\}$, then for any initial data with negative initial energy, the solution blows up at finite time in $L^{p+2}$ norm.

In 2007, Salim A. Messaoudi and Belkacern Said Houari [8] improve Li's result and showed that certain solutions with positive initial energy also blow up in finite time.

Qingyong Gao, Fushan Li, Yanguo Wang [9] obtained the local existence of the solution to the homogeneous Dirichlet boundary value problem for the higher-order nonlinear Kirchhoff-type equation:

$$
u_{t t}+M\left(\left\|D^{m} u(t)\right\|_{2}^{2}\right)(-\Delta)^{m} u+\left|u_{t}\right|^{q-2} u_{t}=|u|^{p-2} u .
$$

where $p>q \geq 2, m \geq 1$.

At present, most Higher-order Kirchhoff-type equations investigate the blow-up of the solution. We study the global attractor of the solution for Higher-order Kirchhofftype equations.

Igor Chueshov [4] studied the longtime dynamics of Kirchhoff wave models with strong nonlinear damping:

$$
u_{t t}-\sigma\left(\|\nabla u\|^{2}\right)(\Delta) u_{t}-\phi\left(\|\nabla u\|^{2}\right)(\Delta) u+f(u)=h(u), x \in \Omega, t>0 .
$$

He proves the existence and uniqueness of weak solutions, and established a finitedimensional global attractor in the sense of partially strong topology.

On the basis of Igor Chueshov, we investigate the global attractor of the higher-order Kirchhoff-type Equation (1.1) with strong nonlinear damping. Such problems have been studied by many authors, but $\sigma\left(\left\|\nabla^{m} u\right\|^{2}\right)$ is a definite constant and even 
$\sigma\left(\left\|\nabla^{m} u\right\|^{2}\right)=0$. Generally, the equation exist a nonlinear $f(u)$. But in the paper, $\sigma\left(\left\|\nabla^{m} u\right\|^{2}\right)$ is a scalar function and $f(u)=0$. Under of the the proper assume, in

section 2, we prove the existence of the solution by priori estimation and the Galerkin method. Therefore, we show that i) the solution $(u, v)$ of the problem (1.1) - (1.3) satisfies $(u, v) \in H_{0}^{m}(\Omega) \times L^{2}(\Omega)$; further more, ii) the solution $(u, v)$ of the problem (1.1) - (1.3) satisfies $(u, v) \in H^{2 m}(\Omega) \times H_{0}^{m}(\Omega)$. Then, in section 3, we prove the uniqueness of the solution by using the method that assumption exist two solutions in the same initial value and two solutions are equal. At last, according to define, we obtain to the existence of the global attractor.

\section{Preliminaries}

For brevity, we denote the simple symbol, $\|\cdot\|$ represents inner product, and $H^{m}=H^{m}(\Omega), \quad H_{0}^{m}=H_{0}^{m}(\Omega), \quad H_{0}^{2 m}=H_{0}^{2 m}(\Omega), \quad H=L^{2},\|\cdot\|=\|\cdot\|_{L^{2}},\|\cdot\|_{\infty}=\|\cdot\|_{L^{\infty}}$, $f=f(x), \quad c_{i}(i=0,1, \cdots, 7)$ are constants, $m_{i}, \mu_{i}(i=0,1)$ are also constants. $\lambda^{m}$ is the first eigenvalue of the operator $\nabla^{m}$.

In this section, we present some assumptions needed in the proof of our results. For this reason, we assume that

$$
\begin{aligned}
\left(\mathrm{H}_{1}\right) \text { setting } \Sigma(s) & =\int_{0}^{s} \sigma(\xi) \mathrm{d} \xi, \Phi(s)=\int_{0}^{s} \phi(s) \mathrm{d} s, \text { then } \\
& s \phi(s)-\varepsilon s \sigma(s)-\eta(\Phi(s)-\Sigma(s))>s,
\end{aligned}
$$

where $\forall \varepsilon>0, \forall \eta>0$.

$\left(\mathrm{H}_{2}\right)[10]$

$$
m_{0}<\phi(s)-\varepsilon \sigma(s)<m_{1}, m=\left\{\begin{array}{l}
m_{0}, \frac{\mathrm{d}}{\mathrm{d} t}\left\|\Delta^{m} u\right\|^{2} \geq 0 \\
m_{1}, \frac{\mathrm{d}}{\mathrm{d} t}\left\|\Delta^{m} u\right\|^{2}<0 .
\end{array}\right.
$$

$\left(\mathrm{H}_{3}\right)$

$$
\sigma(s), \phi(s) \in C^{1}(\Omega)
$$

$\left(\mathrm{H}_{4}\right)$

$$
\mu_{0}<\phi(s)+\varepsilon \sigma(s)<\mu_{1}, \mu=\left\{\begin{array}{l}
\mu_{0}, \frac{\mathrm{d}}{\mathrm{d} t}\left\|\nabla^{m} w\right\|^{2} \geq 0 \\
\mu_{1}, \frac{\mathrm{d}}{\mathrm{d} t}\left\|\nabla^{m} w\right\|^{2}<0 .
\end{array}\right.
$$

Now, we can do priori estimates for equation (1.1)

Lemma 1. Assume $\left(\mathrm{H}_{1}\right)$ hold, and $\left(u_{0}, u_{1}\right) \in H^{m} \times H, f \in H$. Then the solution $(u, v)$ of the problem (1.1) - (1.3) satisfies $(u, v) \in H^{m} \times H$, and

$$
\|(u, v)\|_{H^{m} \times H}^{2}=\left\|\nabla^{m} u\right\|^{2}+\|v\|^{2} \leq W_{1}(0) \mathrm{e}^{-\gamma_{1} t}+\frac{C_{1}}{\gamma_{1}}\left(1-\mathrm{e}^{-\gamma_{1} t}\right),
$$

where $v=u_{t}+\varepsilon u, \quad W_{1}(0)=\left\|v_{0}\right\|^{2}+2 \Phi\left(\left\|\nabla^{m} u_{0}\right\|^{2}\right)-2 \varepsilon \Sigma\left(\left\|\nabla^{m} u_{0}\right\|^{2}\right), \quad v_{0}=u_{1}+\varepsilon u_{0}$. Thus, 
there exists $R_{1}$ and $t=t_{1}>0$, such that

$$
\varlimsup_{t \rightarrow \infty}\|(u, v)\|^{2} \leq \frac{c_{1}}{\gamma_{1}}=R_{1} .
$$

Proof. Let $v=u_{t}+\varepsilon u$, then we use $v$ multiply with both sides of Equation (1.1) and obtain

$$
\left(u_{t t}+\sigma\left(\left\|\nabla^{m} u\right\|^{2}\right)(-\Delta)^{m} u_{t}+\phi\left(\left\|\nabla^{m} u\right\|^{2}\right)(-\Delta)^{m} u, v\right)=(f(x), v) .
$$

After a computation (2.7) one by one, as follow

$$
\begin{aligned}
& \left(u_{t t}, v\right)=\left(v_{t}-\varepsilon u_{t}, v\right)=\left(v_{t}, v\right)-\varepsilon\left(u_{t}, v\right)=\left(v_{t}, v\right)-\varepsilon(v-\varepsilon u, v) \\
& \geq \frac{1}{2} \frac{\mathrm{d}}{\mathrm{d} t}\|v\|^{2}-\varepsilon\|v\|^{2}-\frac{\varepsilon^{2}}{2 \lambda^{m}}\left\|\nabla^{m} u\right\|^{2}-\frac{\varepsilon^{2}}{2}\|v\|^{2} . \\
& \left(\sigma\left(\left\|\nabla^{m} u\right\|^{2}\right)(-\Delta)^{m} u_{t}, v\right) \\
& =\left(\sigma\left(\left\|\nabla^{m} u\right\|^{2}\right)(-\Delta)^{m}(v-\varepsilon u), v\right) \\
& =\left(\sigma\left(\left\|\nabla^{m} u\right\|^{2}\right)(-\Delta)^{m} v, v\right)-\varepsilon\left(\sigma\left(\left\|\nabla^{m} u\right\|^{2}\right)(-\Delta)^{m} u, v\right) \\
& =\sigma\left(\left\|\nabla^{m} u\right\|^{2}\right)\left\|\nabla^{m} v\right\|^{2}-\varepsilon\left(\sigma\left(\left\|\nabla^{m} u\right\|^{2}\right)(-\Delta)^{m} u, u_{t}+\varepsilon u\right) \\
& =\sigma\left(\left\|\nabla^{m} u\right\|^{2}\right)\left\|\nabla^{m} v\right\|^{2}-\frac{\mathrm{d}}{\mathrm{d} t} \varepsilon \Sigma\left(\left\|\nabla^{m} u\right\|^{2}\right)-\varepsilon^{2} \sigma\left(\left\|\nabla^{m} u\right\|^{2}\right)\left\|\nabla^{m} u\right\|^{2} \\
& \geq \lambda^{m} \sigma\left(\left\|\nabla^{m} u\right\|^{2}\right)\|v\|^{2} \frac{\mathrm{d}}{\mathrm{d} t} \varepsilon \Sigma\left(\left\|\nabla^{m} u\right\|^{2}\right)-\varepsilon^{2} \sigma\left(\left\|\nabla^{m} u\right\|^{2}\right)\left\|\nabla^{m} u\right\|^{2} . \\
& \quad\left(\phi\left(\left\|\nabla^{m} u\right\|^{2}\right)(-\Delta)^{m} u, v\right) \\
& =\left(\phi\left(\left\|\nabla^{m} u\right\|^{2}\right)(-\Delta)^{m} u, u_{t}+\varepsilon u\right) \\
& =\left(\phi\left(\left\|\nabla^{m} u\right\|^{2}\right)(-\Delta)^{m} u, u_{t}\right)+\varepsilon\left(\phi\left(\left\|\nabla^{m} u\right\|^{2}\right)(-\Delta)^{m} u, u\right) \\
& =\frac{\mathrm{d}}{\mathrm{d} t} \Phi\left(\left\|\nabla^{m} u\right\|^{2}\right) \varepsilon \phi\left(\left\|\nabla^{m} u\right\|^{2}\right)\left\|\nabla^{m} u\right\|^{2} .
\end{aligned}
$$

Because $f \in H$, by using Holder inequality, Young's inequality, we obtain

$$
(f(x), v) \leq\|f\|\|v\| \leq \frac{1}{2 \varepsilon^{2}}\|f\|^{2}+\frac{\varepsilon^{2}}{2}\|v\|^{2} .
$$

From the above, we have

$$
\begin{aligned}
& \frac{\mathrm{d}}{\mathrm{d} t}\left(\|v\|^{2}+2 \Phi\left(\left\|\nabla^{m} u\right\|^{2}\right)-2 \varepsilon \Sigma\left(\left\|\nabla^{m} u\right\|^{2}\right)\right) \\
& +\left(2 \lambda^{m} \sigma\left(\left\|\nabla^{m} u\right\|^{2}\right)-2 \varepsilon-2 \varepsilon^{2}\right)\|v\|^{2} \\
& +\left(2 \varepsilon \phi\left(\left\|\nabla^{m} u\right\|^{2}\right)-2 \varepsilon^{2} \sigma\left(\left\|\nabla^{m} u\right\|^{2}\right)-\frac{\varepsilon^{2}}{\lambda^{m}}\right)\left\|\nabla^{m} u\right\|^{2} \leq \frac{1}{\varepsilon^{2}}\|f\|^{2} .
\end{aligned}
$$

According to (2.1), we have 


$$
\begin{aligned}
& \left(2 \varepsilon \phi\left(\left\|\nabla^{m} u\right\|^{2}\right)-2 \varepsilon^{2} \sigma\left(\left\|\nabla^{m} u\right\|^{2}\right)-\frac{\varepsilon^{2}}{\lambda^{m}}\right)\left\|\nabla^{m} u\right\|^{2} \\
& \geq\left(2 \varepsilon \phi\left(\left\|\nabla^{m} u\right\|^{2}\right)-2 \varepsilon^{2} \sigma\left(\left\|\nabla^{m} u\right\|^{2}\right)-2 \varepsilon\right)\left\|\nabla^{m} u\right\|^{2} \\
& \geq 2 \varepsilon \eta\left(\Phi\left(\left\|\nabla^{m} u\right\|^{2}\right)-\Sigma\left(\left\|\nabla^{m} u\right\|^{2}\right)\right)
\end{aligned}
$$

where $\varepsilon<2 \lambda^{m}$.

Substitution (2.13) into (2.12), we receive

$$
\begin{aligned}
& \frac{\mathrm{d}}{\mathrm{d} t}\left(\|v\|^{2}+2 \Phi\left(\left\|\nabla^{m} u\right\|^{2}\right)-2 \varepsilon \Sigma\left(\left\|\nabla^{m} u\right\|^{2}\right)\right) \\
& +\left(2 \lambda^{m} \sigma\left(\left\|\nabla^{m} u\right\|^{2}\right)-2 \varepsilon-2 \varepsilon^{2}\right)\|v\|^{2} \\
& +2 \varepsilon \eta\left(\Phi\left(\left\|\nabla^{m} u\right\|^{2}\right)-\Sigma\left(\left\|\nabla^{m} u\right\|^{2}\right)\right) \leq \frac{1}{\varepsilon^{2}}\|f\|^{2} .
\end{aligned}
$$

We deal with the items, we have

$$
\begin{aligned}
& \left(2 \lambda^{m} \sigma\left(\left\|\nabla^{m} u\right\|^{2}\right)-2 \varepsilon-2 \varepsilon^{2}\right)\|v\|^{2}+2 \varepsilon \eta\left(\Phi\left(\left\|\nabla^{m} u\right\|^{2}\right)-\Sigma\left(\left\|\nabla^{m} u\right\|^{2}\right)\right) \\
= & \left(2 \lambda^{m} \sigma\left(\left\|\nabla^{m} u\right\|^{2}\right)-2 \varepsilon-2 \varepsilon^{2}\right)\|v\|^{2}+2 \varepsilon \eta \Phi\left(\left\|\nabla^{m} u\right\|^{2}\right)-\varepsilon^{2} \eta \Sigma\left(\left\|\nabla^{m} u\right\|^{2}\right) \\
& +\varepsilon^{2} \eta \Sigma\left(\left\|\nabla^{m} u\right\|^{2}\right)-2 \varepsilon \eta \Sigma\left(\left\|\nabla^{m} u\right\|^{2}\right) \\
= & \left(2 \lambda^{m} \sigma\left(\left\|\nabla^{m} u\right\|^{2}\right)-2 \varepsilon-2 \varepsilon^{2}\right)\|v\|^{2}+\varepsilon \eta\left(2 \Phi\left(\left\|\nabla^{m} u\right\|^{2}\right)-\varepsilon \Sigma\left(\left\|\nabla^{m} u\right\|^{2}\right)\right) \\
& +\left(\varepsilon^{2} \eta-2 \varepsilon \eta\right) \Sigma\left(\left\|\nabla^{m} u\right\|^{2}\right) \\
\geq & \gamma_{1}\left(\|v\|^{2}+2 \Phi\left(\left\|\nabla^{m} u\right\|^{2}\right)-\varepsilon \Sigma\left(\left\|\nabla^{m} u\right\|^{2}\right)\right)-\gamma_{1} \varepsilon \Sigma\left(\left\|\nabla^{m} u\right\|^{2}\right) \\
= & \gamma_{1}\left(\|v\|^{2}+2 \Phi\left(\left\|\nabla^{m} u\right\|^{2}\right)-2 \varepsilon \Sigma\left(\left\|\nabla^{m} u\right\|^{2}\right)\right),
\end{aligned}
$$

where we take a proper constant $\varepsilon$, such that

$$
\begin{aligned}
& 2 \lambda^{m} \sigma\left(\left\|\nabla^{m} u\right\|^{2}\right)-2 \varepsilon-2 \varepsilon^{2}>0, \gamma_{1}=\min \left\{2 \lambda^{m} \sigma\left(\left\|\nabla^{m} u\right\|^{2}\right)-2 \varepsilon-2 \varepsilon^{2}, \varepsilon \eta\right\}, \\
& \eta=\frac{\gamma_{1}}{2-\varepsilon} .
\end{aligned}
$$

Then, we get

$$
\frac{\mathrm{d}}{\mathrm{d} t} W_{1}(t)+\gamma_{1} W_{1}(t) \leq c_{1}
$$

where

$$
\begin{aligned}
& W_{1}(t)=\|v\|^{2}+2 \Phi\left(\left\|\nabla^{m} u\right\|^{2}\right)-2 \varepsilon \Sigma\left(\left\|\nabla^{m} u\right\|^{2}\right), \\
& C_{1}=\frac{1}{\varepsilon^{2}}\|f\|^{2} .
\end{aligned}
$$

By using Gronwall inequality, we obtain 


$$
W_{1}(t) \leq W_{1}(0) \mathrm{e}^{-\gamma_{1} t}+\frac{c_{1}}{\gamma_{1}}\left(1-\mathrm{e}^{-\gamma_{1} t}\right)
$$

where

$$
\begin{aligned}
& W_{1}(0)=\left\|v_{0}\right\|^{2}+2 \Phi\left(\left\|\nabla^{m} u_{0}\right\|^{2}\right)-2 \varepsilon \Sigma\left(\left\|\nabla^{m} u_{0}\right\|^{2}\right), \\
& v_{0}=u_{1}+\varepsilon u_{0} .
\end{aligned}
$$

So, we have

$$
\|(u, v)\|_{H^{m} \times H}^{2}=\left\|\nabla^{m} u\right\|^{2}+\|v\|^{2} \leq W_{1}(0) \mathrm{e}^{-\gamma_{1} t}+\frac{C_{1}}{\gamma_{1}}\left(1-\mathrm{e}^{-\gamma_{1} t}\right),
$$

and

$$
\varlimsup_{t \rightarrow \infty}\|(u, v)\|^{2} \leq \frac{c_{1}}{\gamma_{1}} .
$$

Thus, there exist $t=t_{1}(\Omega)$ and $t=t_{1}(\Omega)$, such that

$$
\|(u, v)\|_{H^{m} \times H}^{2} \leq \frac{c_{1}}{\gamma_{1}}=R_{1}\left(t>t_{1}\right) .
$$

Remark 1. Assumption $\left(\mathrm{H}_{1}\right)$ imply

$$
\Phi\left(\left\|\nabla^{m} u\right\|^{2}\right)>\varepsilon \Sigma\left(\left\|\nabla^{m} u\right\|^{2}\right)>\varepsilon\left\|\nabla^{m} u\right\|^{2}+c_{0},
$$

such that (2.20) hold.

Lemma 2. Assume $\left(\mathrm{H}_{2}\right)$ hold, $f \in H_{0}^{m}$, and $\left(u_{0}, u_{1}\right) \in H^{2 m} \times H_{0}^{m}$. Then the solution $(u, v)$ of the problem (1.1) - (1.3) satisfies $(u, v) \in H^{2 m} \times H_{0}^{m}$, and

$$
\left\|\nabla^{m} v\right\|^{2}+\left\|\Delta^{m} u\right\|^{2} \leq \frac{W_{2}(0)}{L} \mathrm{e}^{-\gamma_{2} t}+\frac{C_{2}}{L \gamma_{2}}\left(1-\mathrm{e}^{-\gamma_{2} t}\right),
$$

where $\quad v=u_{t}+\varepsilon u, \quad 0<L<\min \{1, m\}, \quad W_{2}(0)=\left\|\nabla^{m} v_{0}\right\|^{2}+m\left\|\Delta^{m} u_{0}\right\|^{2}, \quad v_{0}=u_{1}+\varepsilon u_{0}$. There exist $t=t_{2}(\Omega)$ and $R_{2}$, such that

$$
\varlimsup_{t \rightarrow \infty}\|(u, v)\|_{H^{2 m} \times H_{0}^{m}}^{2} \leq \frac{C_{2}}{L \gamma_{2}}=R_{2} .
$$

Proof. Let $(-\Delta)^{m} v=(-\Delta)^{m} u_{t}+\varepsilon(-\Delta)^{m} u$, we use $(-\Delta)^{m} v$ multiply sides of equation (1.1) and obtain

$$
\left(u_{t t}+\sigma\left(\left\|\nabla^{m} u\right\|^{2}\right)(-\Delta)^{m} u_{t}+\phi\left(\left\|\nabla^{m} u\right\|^{2}\right)(-\Delta)^{m} u,(-\Delta)^{m} v\right)=\left(f(x),(-\Delta)^{m} v\right) \text {. }
$$

After a computation (2.26) one by one, as follow

$$
\begin{aligned}
\left(u_{t t},(-\Delta)^{m} v\right) & =\left(v_{t}-\varepsilon u_{t},(-\Delta)^{m} v\right) \\
& =\frac{1}{2} \frac{\mathrm{d}}{\mathrm{d} t}\left\|\nabla^{m} v\right\|^{2}-\varepsilon\left(v-\varepsilon u,(-\Delta)^{m} v\right) \\
& =\frac{1}{2} \frac{\mathrm{d}}{\mathrm{d} t}\left\|\nabla^{m} v\right\|^{2}-\varepsilon\left\|\nabla^{m} v\right\|^{2}+\varepsilon^{2}\left(u,(-\Delta)^{m} v\right) \\
& \geq \frac{1}{2} \frac{\mathrm{d}}{\mathrm{d} t}\left\|\nabla^{m} v\right\|^{2}-\varepsilon\left\|\nabla^{m} v\right\|^{2}-\frac{\varepsilon^{2}}{2 \lambda^{m}}\left\|\Delta^{m} u\right\|^{2}-\frac{\varepsilon^{2}}{2}\left\|\nabla^{m} v\right\|^{2} .
\end{aligned}
$$




$$
\begin{aligned}
& \left(\sigma\left(\left\|\nabla^{m} u\right\|^{2}\right)(-\Delta)^{m} u_{t},(-\Delta)^{m} v\right) \\
& =\left(\sigma\left(\left\|\nabla^{m} u\right\|^{2}\right)(-\Delta)^{m} v-\varepsilon(-\Delta)^{m} u,(-\Delta)^{m} v\right) \\
& =\sigma\left(\left\|\nabla^{m} u\right\|^{2}\right)\left\|\Delta^{m} v\right\|^{2}-\left(\sigma\left(\left\|\nabla^{m} u\right\|^{2}\right) \varepsilon(-\Delta)^{m} u,(-\Delta)^{m} u_{t}+\varepsilon(-\Delta)^{m} u\right) \\
& =\sigma\left(\left\|\nabla^{m} u\right\|^{2}\right)\left\|\Delta^{m} v\right\|^{2}-\frac{1}{2} \varepsilon \sigma\left(\left\|\nabla^{m} u\right\|^{2}\right) \frac{\mathrm{d}}{\mathrm{d} t}\left\|\Delta^{m} u\right\|^{2}-\varepsilon^{2} \sigma\left(\left\|\nabla^{m} u\right\|^{2}\right)\left\|\Delta^{m} u\right\|^{2} \\
& \geq \lambda^{m} \sigma\left(\left\|\nabla^{m} u\right\|^{2}\right)\left\|\nabla^{m} v\right\|^{2}-\frac{1}{2} \varepsilon \sigma\left(\left\|\nabla^{m} u\right\|^{2}\right) \frac{\mathrm{d}}{\mathrm{d} t}\left\|\Delta^{m} u\right\|^{2}-\varepsilon^{2} \sigma\left(\left\|\nabla^{m} u\right\|^{2}\right)\left\|\Delta^{m} u\right\|^{2} . \\
& \quad\left(\phi\left(\left\|\nabla^{m} u\right\|^{2}\right)(-\Delta)^{m} u,(-\Delta)^{m} v\right) \\
& =\left(\phi\left(\left\|\nabla^{m} u\right\|^{2}\right)(-\Delta)^{m} u,(-\Delta)^{m} u_{t}+\varepsilon(-\Delta)^{m} u\right) \\
& =\frac{1}{2} \phi\left(\left\|\nabla^{m} u\right\|^{2}\right) \frac{\mathrm{d}}{\mathrm{d} t}\left\|\Delta^{m} u\right\|^{2}+\varepsilon \phi\left(\left\|\nabla^{m} u\right\|^{2}\right)\left\|\Delta^{m} u\right\|^{2} .
\end{aligned}
$$

Due to $f \in H_{0}^{m}$, by using Holder inequality, Young's inequality, we obtain

$$
\left(f(x),(-\Delta)^{m} v\right) \leq \frac{\varepsilon^{2}}{2}\left\|\nabla^{m} v\right\|^{2}+\frac{1}{2 \varepsilon^{2}}\left\|\nabla^{m} f\right\|^{2} .
$$

From the above, we obtain

$$
\begin{aligned}
& \frac{\mathrm{d}}{\mathrm{d} t}\left\|\nabla^{m} v\right\|^{2}+\left(\phi\left(\left\|\nabla^{m} u\right\|^{2}\right)-\varepsilon \sigma\left(\left\|\nabla^{m} u\right\|^{2}\right)\right) \frac{\mathrm{d}}{\mathrm{d} t}\left\|\Delta^{m} u\right\|^{2} \\
& +\left(2 \lambda^{m} \sigma\left(\left\|\nabla^{m} u\right\|^{2}\right)-2 \varepsilon-2 \varepsilon^{2}\right)\left\|\nabla^{m} v\right\|^{2} \\
& +\left(2 \varepsilon \phi\left(\left\|\nabla^{m} u\right\|^{2}\right)-2 \varepsilon^{2} \sigma\left(\left\|\nabla^{m} u\right\|^{2}\right)-\frac{\varepsilon^{2}}{\lambda^{m}}\right)\left\|\Delta^{m} u\right\|^{2} \leq \frac{1}{\varepsilon^{2}}\left\|\nabla^{m} f\right\|^{2} .
\end{aligned}
$$

According to (2.2), we have

$$
\begin{aligned}
& \left(\phi\left(\left\|\nabla^{m} u\right\|^{2}\right)-\varepsilon \sigma\left(\left\|\nabla^{m} u\right\|^{2}\right)\right) \frac{\mathrm{d}}{\mathrm{d} t}\left\|\Delta^{m} u\right\|^{2} \\
& +\left(2 \varepsilon \phi\left(\left\|\nabla^{m} u\right\|^{2}\right)-2 \varepsilon^{2} \sigma\left(\left\|\nabla^{m} u\right\|^{2}\right)-\frac{\varepsilon^{2}}{\lambda^{m}}\right)\left\|\Delta^{m} u\right\|^{2} \\
& \geq m \frac{\mathrm{d}}{\mathrm{d} t}\left\|\Delta^{m} u\right\|^{2}+\left(2 \varepsilon m_{0}-\frac{\varepsilon^{2}}{\lambda^{m}}\right)\left\|\Delta^{m} u\right\|^{2} .
\end{aligned}
$$

Collecting with (2.32), we obtain from (2.31) that

$$
\begin{aligned}
& \frac{\mathrm{d}}{\mathrm{d} t}\left(\left\|\nabla^{m} v\right\|^{2}+m\left\|\Delta^{m} u\right\|^{2}\right)+\left(2 \lambda^{m} \sigma\left(\left\|\nabla^{m} u\right\|^{2}\right)-2 \varepsilon-2 \varepsilon^{2}\right)\left\|\nabla^{m} v\right\|^{2} \\
& +\left(2 \varepsilon m_{0}-\frac{\varepsilon^{2}}{\lambda^{m}}\right)\left\|\Delta^{m} u\right\|^{2} \leq \frac{1}{\varepsilon^{2}}\left\|\nabla^{m} f\right\|^{2} .
\end{aligned}
$$

Noticing $0<\varepsilon<\lambda^{m}\left(2 m_{0}-m\right)$, this will imply

$$
\left(2 \varepsilon m_{0}-\frac{\varepsilon^{2}}{\lambda^{m}}\right)\left\|\Delta^{m} u\right\|^{2} \geq \varepsilon m\left\|\Delta^{m} u\right\|^{2} .
$$


Substituting (2.34) into (2.33), we can get the following inequality

$$
\begin{aligned}
& \frac{\mathrm{d}}{\mathrm{d} t}\left(\left\|\nabla^{m} v\right\|^{2}+m\left\|\Delta^{m} u\right\|^{2}\right)+\left(2 \lambda^{m} \sigma\left(\left\|\nabla^{m} u\right\|^{2}\right)-2 \varepsilon-2 \varepsilon^{2}\right)\left\|\nabla^{m} v\right\|^{2} \\
& +\varepsilon m\left\|\Delta^{m} u\right\|^{2} \leq \frac{1}{\varepsilon^{2}}\left\|\nabla^{m} f\right\|^{2} .
\end{aligned}
$$

Hence, we take a proper constant $\varepsilon$, such that $2 \lambda^{m} \sigma\left(\left\|\nabla^{m} u\right\|^{2}\right)-2 \varepsilon-2 \varepsilon^{2}>0$, we get

$$
\frac{\mathrm{d}}{\mathrm{d} t} W_{2}(t)+\gamma_{2} W_{2}(t) \leq c_{2}
$$

where

$$
\begin{aligned}
& W_{2}(t)=\left\|\nabla^{m} v\right\|^{2}+m\left\|\Delta^{m} u\right\|^{2}, \\
& \gamma_{2}=\min \left\{2 \lambda^{m} \sigma\left(\left\|\nabla^{m} u\right\|^{2}\right)-2 \varepsilon-2 \varepsilon^{2}, \varepsilon\right\}, \\
& c_{2}=\frac{1}{\varepsilon^{2}}\left\|\nabla^{m} f\right\|^{2} .
\end{aligned}
$$

By using Gronwall inequality, we end up with

$$
W_{2}(t) \leq W_{2}(0) \mathrm{e}^{-\gamma_{2} t}+\frac{c_{2}}{\gamma_{2}}\left(1-\mathrm{e}^{-\gamma_{2} t}\right),
$$

where

$$
\begin{aligned}
& W_{2}(0)=\left\|\nabla^{m} v_{0}\right\|^{2}+m\left\|\Delta^{m} u_{0}\right\|^{2}, \\
& v_{0}=u_{1}+\varepsilon u_{0},
\end{aligned}
$$

Taking $L=\min \{1, m\}$, we have

$$
\left\|\nabla^{m} v\right\|^{2}+\left\|\Delta^{m} u\right\|^{2} \leq \frac{W_{2}(0)}{L} \mathrm{e}^{-\gamma_{2} t}+\frac{C_{2}}{L \gamma_{2}}\left(1-\mathrm{e}^{-\gamma_{2} t}\right),
$$

and

$$
\varlimsup_{t \rightarrow \infty}\|(u, v)\|_{H^{2 m} \times H_{0}^{m}}^{2} \leq \frac{C_{2}}{L \gamma_{2}} .
$$

Thus, there exist $t=t_{2}(\Omega)$ and $R_{2}$, such that

$$
\|(u, v)\|_{H^{2 m} \times H_{0}^{m}}^{2} \leq R_{2}\left(t>t_{2}\right) .
$$

\section{Global Attractor}

\subsection{The Existence and Uniqueness of Solution}

Theorem 3.1. Assume $\left(\mathrm{H}_{1}\right)-\left(\mathrm{H}_{4}\right)$ hold, and $\left(u_{0}, u_{1}\right) \in H^{2 m} \times H_{0}^{m}, f(x) \in H_{0}^{m}$, $v=u_{t}+\varepsilon u$. So equality (1.1) exists a unique smooth solution $(u, v) \in L^{\infty}\left((0,+\infty), H^{2 m} \times H_{0}^{m}\right)$.

Remark 2. We denote the solution in Theorem 3.1 by $S(t)\left(u_{0}, u_{1}\right)=\left(u(t), u_{t}(t)\right)$. Then $S(t)$ composes a continuous semigroup in $H^{2 m} \times H_{0}^{m}$.

Proof. By the Galerkin method, Lemma 1 and Lemma 2, we can easily obtain the existence of Solutions, the procedure is omitted. Next, we prove the uniqueness of Solu- 
tions in detail. Let $u, v$ are two solutions of the problems (1.1) - (1.3), we denote $w=u-v$, then $w(x, 0)=w_{0}(x)=0, w_{t}(x, 0)=w_{1}(x)=0$ and the two equations subtract and obtain

$$
\begin{aligned}
& w_{t t}+\sigma\left(\left\|\nabla^{m} u\right\|^{2}\right)(-\Delta)^{m} u_{t}-\sigma\left(\left\|\nabla^{m} v\right\|^{2}\right)(-\Delta)^{m} v_{t} \\
& +\phi\left(\left\|\nabla^{m} u\right\|^{2}\right)(-\Delta)^{m} u-\phi\left(\left\|\nabla^{m} v\right\|^{2}\right)(-\Delta)^{m} v=0 .
\end{aligned}
$$

By using $w_{t}+\varepsilon w$ to inner product of the equation (3.1), and we have

$$
\begin{gathered}
\left(w_{t t}+\sigma\left(\left\|\nabla^{m} u\right\|^{2}\right)(-\Delta)^{m} u_{t}-\sigma\left(\left\|\nabla^{m} v\right\|^{2}\right)(-\Delta)^{m} v_{t}\right. \\
\left.+\phi\left(\left\|\nabla^{m} u\right\|^{2}\right)(-\Delta)^{m} u-\phi\left(\left\|\nabla^{m} v\right\|^{2}\right)(-\Delta)^{m} v, w_{t}+\varepsilon w\right) \\
\left(w_{t t}, w_{t}+\varepsilon w\right)=\left(w_{t t}, w_{t}\right)+\varepsilon\left(w_{t t}, w\right) \\
=\frac{1}{2} \frac{\mathrm{d}}{\mathrm{d} t}\left\|w_{t}\right\|^{2}+\varepsilon \frac{\mathrm{d}}{\mathrm{d} t}\left(w_{t}, w\right)-\varepsilon\left\|w_{t}\right\|^{2} . \\
\left(\sigma\left(\left\|\nabla^{m} u\right\|^{2}\right)(-\Delta)^{m} u_{t}-\sigma\left(\left\|\nabla^{m} v\right\|^{2}\right)(-\Delta)^{m} v_{t}, w_{t}+\varepsilon w\right) \\
=\left(\sigma\left(\left\|\nabla^{m} u\right\|^{2}\right)(-\Delta)^{m} u_{t}-\sigma\left(\left\|\nabla^{m} v\right\|^{2}\right)(-\Delta)^{m} v_{t}, w_{t}\right) \\
\quad+\varepsilon\left(\sigma\left(\left\|\nabla^{m} u\right\|^{2}\right)(-\Delta)^{m} u_{t}-\sigma\left(\left\|\nabla^{m} v\right\|^{2}\right)(-\Delta)^{m} v_{t}, w\right) \\
:=I_{1}+\varepsilon I_{2},
\end{gathered}
$$

Next, we process each item in turn

$$
\begin{aligned}
I_{1}= & \left(\sigma\left(\left\|\nabla^{m} u\right\|^{2}\right)(-\Delta)^{m} u_{t}-\sigma\left(\left\|\nabla^{m} u\right\|^{2}\right)(-\Delta)^{m} v_{t}\right. \\
& \left.+\sigma\left(\left\|\nabla^{m} u\right\|^{2}\right)(-\Delta)^{m} v_{t}-\sigma\left(\left\|\nabla^{m} v\right\|^{2}\right)(-\Delta)^{m} v_{t}, w_{t}\right) \\
= & \sigma\left(\left\|\nabla^{m} u\right\|^{2}\right)\left((-\Delta)^{m} w_{t}, w_{t}\right)+\left(\sigma\left(\left\|\nabla^{m} u\right\|^{2}\right)-\sigma\left(\left\|\nabla^{m} v\right\|^{2}\right)\right)\left((-\Delta)^{m} v_{t}, w_{t}\right) \\
= & \sigma\left(\left\|\nabla^{m} u\right\|^{2}\right)\left\|\nabla^{m} w_{t}\right\|^{2}+\sigma^{\prime}(\xi)\left(\left\|\nabla^{m} u\right\|+\left\|\nabla^{m} v\right\|\right)\left(\left\|\nabla^{m} u\right\|-\left\|\nabla^{m} v\right\|\right)\left(\nabla^{m} v_{t}, \nabla^{m} w_{t}\right) \\
\geq & \sigma\left(\left\|\nabla^{m} u\right\|^{2}\right)\left\|\nabla^{m} w_{t}\right\|^{2}-\left|\sigma^{\prime}(\xi)\right|\left(\left\|\nabla^{m} u\right\|+\left\|\nabla^{m} v\right\|\right)\left\|\nabla^{m} w\right\|\left\|\nabla^{m} v_{t}\right\|\left\|\nabla^{m} w_{t}\right\| \\
\geq & \sigma\left(\left\|\nabla^{m} u\right\|^{2}\right)\left\|\nabla^{m} w_{t}\right\|^{2}-\left\|\sigma^{\prime}(\xi)\right\|\left\|_{\infty}\left(\left\|\nabla^{m} u\right\|+\left\|\nabla^{m} v\right\|\right)\right\| \nabla^{m} w\|\| \nabla^{m} v_{t}\|\| \nabla^{m} w_{t} \| \\
= & \sigma\left(\left\|\nabla^{m} u\right\|^{2}\right)\left\|\nabla^{m} w_{t}\right\|^{2}-c_{3}\left\|\nabla^{m} w\right\|\left\|\nabla^{m} w_{t}\right\| \\
\geq & \sigma\left(\left\|\nabla^{m} u\right\|^{2}\right)\left\|\nabla^{m} w_{t}\right\|^{2}-\frac{\varepsilon}{2}\left\|\nabla^{m} w\right\|^{2}-\frac{c_{3}^{2}}{2 \varepsilon}\left\|\nabla^{m} w_{t}\right\|^{2} \\
= & \left(\sigma\left(\left\|\nabla^{m} u\right\|^{2}\right)-\frac{c_{3}^{2}}{2 \varepsilon}\right)\left\|\nabla^{m} w_{t}\right\|^{2}-\frac{\varepsilon}{2}\left\|\nabla^{m} w\right\|^{2} .
\end{aligned}
$$

Analogous to $I_{1}$, we deal with $I_{2}$ 


$$
\begin{aligned}
I_{2}= & \left(\sigma\left(\left\|\nabla^{m} u\right\|^{2}\right)(-\Delta)^{m} u_{t}-\sigma\left(\left\|\nabla^{m} u\right\|^{2}\right)(-\Delta)^{m} v_{t}\right. \\
& \left.+\sigma\left(\left\|\nabla^{m} u\right\|^{2}\right)(-\Delta)^{m} v_{t}-\sigma\left(\left\|\nabla^{m} v\right\|^{2}\right)(-\Delta)^{m} v_{t}, w\right) \\
= & \frac{\sigma\left(\left\|\nabla^{m} u\right\|^{2}\right)}{2} \frac{\mathrm{d}}{\mathrm{d} t}\left\|\nabla^{m} w\right\|^{2}+\sigma^{\prime}(\xi)\left(\left\|\nabla^{m} u\right\|+\left\|\nabla^{m} v\right\|\right)\left(\left\|\nabla^{m} u\right\|-\left\|\nabla^{m} v\right\|\right)\left((-\Delta)^{m} v_{t}, w\right) \\
\geq & \frac{\sigma\left(\left\|\nabla^{m} u\right\|^{2}\right)}{2} \frac{\mathrm{d}}{\mathrm{d} t}\left\|\nabla^{m} w\right\|^{2}-\left|\sigma^{\prime}(\xi)\right|\left(\left\|\nabla^{m} u\right\|+\left\|\nabla^{m} v\right\|\right)\left\|\nabla^{m} v_{t}\right\|\left\|\nabla^{m} w\right\|^{2} \\
= & \frac{\sigma\left(\left\|\nabla^{m} u\right\|^{2}\right)}{2} \frac{\mathrm{d}}{\mathrm{d} t}\left\|\nabla^{m} w\right\|^{2}-c_{4}\left\|\nabla^{m} w\right\|^{2} .
\end{aligned}
$$

Combining with (3.5) - (3.6), we obtain from (3.4) that

$$
\begin{aligned}
& \left(\sigma\left(\left\|\nabla^{m} u\right\|^{2}\right)(-\Delta)^{m} u_{t}-\sigma\left(\left\|\nabla^{m} v\right\|^{2}\right)(-\Delta)^{m} v_{t}, w_{t}+\varepsilon w\right) \\
& \geq\left(\sigma\left(\left\|\nabla^{m} u\right\|^{2}\right)-\frac{c_{3}{ }^{2}}{2 \varepsilon}\right)\left\|\nabla^{m} w_{t}\right\|^{2}-\frac{\varepsilon}{2}\left\|\nabla^{m} w\right\|^{2}+\frac{\varepsilon}{2} \sigma\left(\left\|\nabla^{m} u\right\|^{2}\right) \frac{\mathrm{d}}{\mathrm{d} t}\left\|\nabla^{m} w\right\|^{2}-\varepsilon c_{4}\left\|\nabla^{m} w\right\|^{2} \\
& =\left(\sigma\left(\left\|\nabla^{m} u\right\|^{2}\right)-\frac{c_{3}^{2}}{2 \varepsilon}\right)\left\|\nabla^{m} w_{t}\right\|^{2}+\frac{\varepsilon}{2} \sigma\left(\left\|\nabla^{m} u\right\|^{2}\right) \frac{\mathrm{d}}{\mathrm{d} t}\left\|\nabla^{m} w\right\|^{2}-\left(\varepsilon c_{4}+\frac{\varepsilon}{2}\right)\left\|\nabla^{m} w\right\|^{2} .
\end{aligned}
$$

Similarly,

$$
\begin{aligned}
& \left(\phi\left(\left\|\nabla^{m} u\right\|^{2}\right)(-\Delta)^{m} u-\phi\left(\left\|\nabla^{m} v\right\|^{2}\right)(-\Delta)^{m} v, w_{t}+\varepsilon w\right) \\
& =\left(\phi\left(\left\|\nabla^{m} u\right\|^{2}\right)(-\Delta)^{m} u-\phi\left(\left\|\nabla^{m} v\right\|^{2}\right)(-\Delta)^{m} v, w_{t}\right) \\
& \quad+\varepsilon\left(\phi\left(\left\|\nabla^{m} u\right\|^{2}\right)(-\Delta)^{m} u-\phi\left(\left\|\nabla^{m} v\right\|^{2}\right)(-\Delta)^{m} v, w\right) \\
& \geq \frac{\phi\left(\left\|\nabla^{m} u\right\|^{2}\right)}{2} \frac{\mathrm{d}}{\mathrm{d} t}\left\|\nabla^{m} w\right\|^{2}-\frac{\varepsilon}{2}\left\|\nabla^{m} w\right\|^{2}-\frac{c_{5}^{2}}{2 \varepsilon}\left\|\nabla^{m} w_{t}\right\|^{2} \\
& \quad+\varepsilon \phi\left(\left\|\nabla^{m} u\right\|^{2}\right)\left\|\nabla^{m} w\right\|^{2}-\varepsilon c_{6}\left\|\nabla^{m} w\right\|^{2} \\
& =\frac{\phi\left(\left\|\nabla^{m} u\right\|^{2}\right)}{2} \frac{\mathrm{d}}{\mathrm{d} t}\left\|\nabla^{m} w\right\|^{2}-\frac{c_{5}^{2}}{2 \varepsilon}\left\|\nabla^{m} w_{t}\right\|^{2}+\left(\varepsilon \phi\left(\left\|\nabla^{m} u\right\|^{2}\right)-\frac{\varepsilon}{2}-\varepsilon c_{6}\right)\left\|\nabla^{m} w\right\|^{2} .
\end{aligned}
$$

Therefore, by the above inequality

$$
\begin{aligned}
& \frac{\mathrm{d}}{\mathrm{d} t}\left(\left\|w_{t}\right\|^{2}+2 \varepsilon\left(w_{t}, w\right)\right)+\left(\phi\left(\left\|\nabla^{m} u\right\|^{2}\right)+\varepsilon \sigma\left(\left\|\nabla^{m} u\right\|^{2}\right)\right) \frac{\mathrm{d}}{\mathrm{d} t}\left\|\nabla^{m} w\right\|^{2} \\
& +\left(2 \sigma\left(\left\|\nabla^{m} u\right\|^{2}\right)-\frac{c_{5}^{2}+c_{3}^{2}}{\varepsilon}\right)\left\|\nabla^{m} w_{t}\right\|^{2}-2 \varepsilon\left\|w_{t}\right\|^{2} \\
& +\left(2 \varepsilon \phi\left(\left\|\nabla^{m} u\right\|^{2}\right)-2 \varepsilon-2 \varepsilon c_{4}-2 \varepsilon c_{6}\right)\left\|\nabla^{m} w\right\|^{2} \leq 0 .
\end{aligned}
$$

when $\sigma\left(\left\|\nabla^{m} u\right\|^{2}\right)>\frac{c_{5}^{2}+c_{3}^{2}}{2 \varepsilon}$, we get 


$$
\begin{aligned}
& \frac{\mathrm{d}}{\mathrm{d} t}\left(\left\|w_{t}\right\|^{2}+2 \varepsilon\left(w_{t}, w\right)\right)+\left(\phi\left(\left\|\nabla^{m} u\right\|^{2}\right)+\varepsilon \sigma\left(\left\|\nabla^{m} u\right\|^{2}\right)\right) \frac{\mathrm{d}}{\mathrm{d} t}\left\|\nabla^{m} w\right\|^{2} \\
& \leq 2 \varepsilon\left\|w_{t}\right\|^{2}+2 \varepsilon\left(1+c_{4}+c_{6}-\phi\left(\left\|\nabla^{m} u\right\|^{2}\right)\right)\left\|\nabla^{m} w\right\|^{2} .
\end{aligned}
$$

In view of $\left(\mathrm{H}_{4}\right)$, there exist constant $\mu$, and let $c_{7}=1+c_{4}+c_{6}$, such that

$$
\frac{\mathrm{d}}{\mathrm{d} t}\left(\left\|w_{t}\right\|^{2}+2 \varepsilon\left(w_{t}, w\right)+\mu\left\|\nabla^{m} w\right\|^{2}\right) \leq 2 \varepsilon\left\|w_{t}\right\|^{2}+2 \varepsilon\left(c_{7}-\phi\left(\left\|\nabla^{m} u\right\|^{2}\right)\right)\left\|\nabla^{m} w\right\|^{2} .
$$

According to Hölder inequality, Young's inequality and Poincaré inequality, we obtain

$$
\varepsilon^{2}\left(w_{t}, w\right) \geq-\frac{\varepsilon^{2}}{2}\left\|w_{t}\right\|^{2}-\frac{\varepsilon^{2}}{2}\|w\|^{2} \geq-\frac{\varepsilon^{2}}{2}\left\|w_{t}\right\|^{2}-\frac{\varepsilon^{2}}{2 \lambda^{m}}\left\|\nabla^{m} w\right\|^{2} .
$$

Combining with (3.11) - (3.12), we receive

$$
\begin{aligned}
& \frac{\mathrm{d}}{\mathrm{d} t}\left(\left\|w_{t}\right\|^{2}+2 \varepsilon\left(w_{t}, w\right)+\mu\left\|\nabla^{m} w\right\|^{2}\right) \\
& \leq\left(2 \varepsilon+\varepsilon^{2}\right)\left\|w_{t}\right\|^{2}+2 \varepsilon\left(c_{7}+\frac{\varepsilon^{2}}{2 \lambda^{m}}-\phi\left(\left\|\nabla^{m} u\right\|^{2}\right)\right)\left\|\nabla^{m} w\right\|^{2}+2 \varepsilon^{2}\left(w_{t}, w\right) \\
& =\varepsilon\left[(2+\varepsilon)\left\|w_{t}\right\|^{2}+2\left(c_{7}+\frac{\varepsilon^{2}}{2 \lambda^{m}}-\phi\left(\left\|\nabla^{m} u\right\|^{2}\right)\right)\left\|\nabla^{m} w\right\|^{2}+2 \varepsilon\left(w_{t}, w\right)\right] .
\end{aligned}
$$

Next, we prove that there is a constant $K$ large enough, such that

$$
\begin{aligned}
& (2+\varepsilon)\left\|w_{t}\right\|^{2}+2\left(c_{7}+\frac{\varepsilon^{2}}{2 \lambda^{m}}-\phi\left(\left\|\nabla^{m} u\right\|^{2}\right)\right)\left\|\nabla^{m} w\right\|^{2}+2 \varepsilon\left(w_{t}, w\right) \\
& \leq K\left(\left\|w_{t}\right\|^{2}+2 \varepsilon\left(w_{t}, w\right)+\mu\left\|\nabla^{m} w\right\|^{2}\right) .
\end{aligned}
$$

Supposing there is a constant $K$ large enough, we have

$$
\begin{aligned}
& (2+\varepsilon-K)\left\|w_{t}\right\|^{2}+2\left(c_{7}+\frac{\varepsilon^{2}}{2 \lambda^{m}}-\phi\left(\left\|\nabla^{m} u\right\|^{2}\right)-\frac{1}{2} K \mu\right)\left\|\nabla^{m} w\right\|^{2}+(2 \varepsilon-2 \varepsilon K)\left(w_{t}, w\right) \\
& \leq(2+\varepsilon-K)\left\|w_{t}\right\|^{2}+2\left(c_{7}+\frac{\varepsilon^{2}}{2 \lambda^{m}}-\phi\left(\left\|\nabla^{m} u\right\|^{2}\right)-\frac{1}{2} K \mu\right)\left\|\nabla^{m} w\right\|^{2}+\left|(2 \varepsilon-2 \varepsilon K)\left(w_{t}, w\right)\right| \\
& \leq(2+\varepsilon-K)\left\|w_{t}\right\|^{2}+2\left(c_{7}+\frac{\varepsilon^{2}}{2 \lambda^{m}}-\phi\left(\left\|\nabla^{m} u\right\|^{2}\right)-\frac{1}{2} K \mu\right)\left\|\nabla^{m} w\right\|^{2}+2 \varepsilon(K-1)\left\|w_{t}\right\|\|w\| \\
& \leq(2+\varepsilon-K)\left\|w_{t}\right\|^{2}+2\left(c_{7}+\frac{\varepsilon^{2}}{2 \lambda^{m}}-\phi\left(\left\|\nabla^{m} u\right\|^{2}\right)-\frac{1}{2} K \mu\right)\left\|\nabla^{m} w\right\|^{2} \\
& \quad+\varepsilon(K-1)\left\|w_{t}\right\|^{2}+\frac{\varepsilon(K-1)}{\lambda^{m}}\left\|\nabla^{m} w\right\|^{2} \\
& =[2+(\varepsilon-1) K]\left\|w_{t}\right\|^{2}+2\left[c_{7}-\phi\left(\left\|\nabla^{m} u\right\|^{2}\right)+\left(\frac{\varepsilon^{2}}{2 \lambda^{m}}-\frac{1}{2} \mu\right) K\right]\left\|\nabla^{m} w\right\|^{2} \\
& \leq 0 \text {, } \\
& \text { where } \varepsilon=\min \left\{1, \lambda^{m} \mu\right\}, \quad \phi\left(\left\|\nabla^{m} u\right\|^{2}\right)<c_{7}+\frac{\varepsilon^{2}}{2 \lambda^{m}} .
\end{aligned}
$$


Hence, there is a constant $K$ large enough, such that (3.14) hold.

Due to (3.14), we have

$$
\frac{\mathrm{d}}{\mathrm{d} t} Y(t) \leq \varepsilon K Y(t)
$$

where

$$
Y(t)=\left\|w_{t}\right\|^{2}+2 \varepsilon\left(w_{t}, w\right)+\mu\left\|\nabla^{m} w\right\|^{2}
$$

Therefore,

$$
0 \leq Y(t) \leq Y(0) \mathrm{e}^{\varepsilon K t}=0
$$

where

$$
Y(0)=\left\|w_{t}(0)\right\|^{2}+2 \varepsilon\left(w_{t}(0), w(0)\right)+\mu\left\|\nabla^{m} w(0)\right\|^{2}
$$

So, we can get

$$
\left\|w_{t}\right\|^{2}+2 \varepsilon\left(w_{t}, w\right)+\mu\left\|\nabla^{m} w\right\|^{2}=0
$$

According to (3.12), we get

$$
(1-\varepsilon)\left\|w_{t}\right\|^{2}+\left(\mu-\frac{\varepsilon}{\lambda^{m}}\right)\left\|\nabla^{m} w\right\|^{2} \leq 0 .
$$

That shows that

$$
\left\|w_{t}\right\|^{2}=0, \quad\left\|\nabla^{m} w\right\|^{2}=0
$$

That is

$$
w(x, t)=0
$$

Therefore,

$$
u=v \text {. }
$$

So we prove the uniqueness of the solution.

\subsection{Global Attractor}

Theorem 3.2. [11] Let $E$ be a Banach space, and $\{S(t)\}(t \geq 0)$ are the semigroup operator on $E$. $S(t): E \rightarrow E, S(t+\tau)=S(t) S(\tau)(\forall t, \tau \geq 0), S(0)=I$, here $I$ is a unit operator. Set $S(t)$ satisfy the follow conditions:

1) $S(t)$ is uniformly bounded, namely $\forall R>0,\|u\|_{E} \leq R$, it exists a constant $C(R)$, so that

$$
\|S(t) u\|_{E} \leq C(R)(t \in[0,+\infty)) ;
$$

2) It exists a bounded absorbing set $B_{0} \subset E$, namely, $\forall B \subset E$, it exists a constant $t_{0}$, so that

$$
S(t) B \subset B_{0}\left(t \geq t_{0}\right)
$$

where $B_{0}$ and $B$ are bounded sets.

3) When $t>0, S(t)$ is a completely continuous operator A. 
Therefore, the semigroup operators $S(t)$ exists a compact global attractor A.

Theorem 3.3. Under the assume of Lemma 1, Lemma 2 and Theorem 3.1, equations have global attractor

$$
A=\omega\left(B_{0}\right)=\bigcap_{\tau \geq 0} \overline{\bigcup_{t \geq \tau} S(t) B_{0}}
$$

where

$$
B_{0}=\left\{(u, v) \in H^{2 m} \times H_{0}^{m}:\|(u, v)\|_{H^{2 m} \times H_{0}^{m}}^{2}=\|u\|_{H^{2 m}}^{2}+\|v\|_{H_{0}^{m}}^{2} \leq R_{1}+R_{2}\right\},
$$

$B_{0}$ is the bounded absorbing set of $H^{2 m} \times H_{0}^{m}$ and satisfies.

1) $S(t) A=A, t>0$;

2) $\lim _{t \rightarrow \infty} \operatorname{dist}(S(t) B, A)=0$, here $B \subset H^{2 m} \times H_{0}^{m}$ and it is a bounded set,

$$
\operatorname{dist}(S(t) B, A)=\sup _{x \in B}\left(\inf _{y \in A}\|S(t) x-y\|_{H^{2 m} \times H_{0}^{m}}\right) \rightarrow 0, t \rightarrow \infty .
$$

Proof. Under the conditions of Theorem 3.1, it exists the solution semigroup S(t), $S(t): H^{2 m} \times H_{0}^{m} \rightarrow H^{2 m} \times H_{0}^{m}$, here $E=H^{2 m} \times H_{0}^{m}$.

1) From Lemma 1 to Lemma 2, we can get that $\forall B \subset H^{2 m} \times H_{0}^{m}$ is a bounded set that includes in the ball $\left\{\|(u, v)\|_{H^{2 m} \times H_{0}^{m}} \leq R\right\}$,

$$
\begin{aligned}
\left\|S(t)\left(u_{0}, v_{0}\right)\right\|_{H^{2 m} \times H_{0}^{m}}^{2} & =\|u\|_{H^{2 m}}^{2}+\|v\|_{H_{0}^{m}}^{2}+C \\
& \leq\left\|u_{0}\right\|_{H^{2 m}}^{2}+\left\|v_{0}\right\|_{H_{0}^{m}}^{2}+C \\
& \leq R^{2}+C, \quad\left(t \geq 0,\left(u_{0}, v_{0}\right) \in B\right)
\end{aligned}
$$

This shows that $S(t)(t \geq 0)$ is uniformly bounded in $H^{2 m} \times H_{0}^{m}$.

2) Furthermore, for any $\left(u_{0}, v_{0}\right) \in H^{2 m} \times H_{0}^{m}$, when $t \geq \max \left\{t_{1}, t_{2}\right\}$, we have

$$
\left\|S(t)\left(u_{0}, v_{0}\right)\right\|_{H^{2 m} \times H_{0}^{m}}^{2}=\|u\|_{H^{2 m}}^{2}+\|v\|_{H_{0}^{m}}^{2} \leq R_{1}+R_{2} .
$$

So we get $B_{0}$ is the bounded absorbing set.

3) Since $H^{2 m} \times H_{0}^{m} \hookrightarrow H^{m} \times H$ is compact embedded, which means that the bounded set in $H^{2 m} \times H^{m}$ is the compact set in $H^{m} \times H$, so the semigroup operator $\mathrm{S}(\mathrm{t})$ exist a compact global attractor $\mathrm{A}$.

The prove is completed.

\section{Conclusion}

The paper's main results deal with global attractors. At first, we prove the existence and uniqueness of the solution. Then we establish the existence of the global attractors. Therefore, we show that i) the solution $(u, v)$ of the problem (1.1) - (1.3) satisfies $(u, v) \in H_{0}^{m}(\Omega) \times L^{2}(\Omega)$; furthermore, ii) the solution $(u, v)$ of the problem (1.1) (1.3) satisfies $(u, v) \in H^{2 m}(\Omega) \times H_{0}^{m}(\Omega)$. Then, we prove the uniqueness of the solution. At last, according to define and theorem, we obtain to the existence of the global attractor.

\section{Acknowledgements}

We express our sincere thanks to the anonymous reviewer for his/her careful reading of 
the paper, we hope that we can get valuable comments and suggestions. These contributions greatly improved the paper, and making the paper better.

\section{Fund}

This work is supported by the National Natural Sciences Foundation of People's Republic of China under Grant 11561076.

\section{References}

[1] Kirchhoff, G. (1883) Vorlesungen fiber Mechanik. Tenbner, Stuttgarty.

[2] Yang, Z.J. and Ding, P.Y. (2016) Longtime Dynamics of the Kirchhoff Equations with Strong Damping and Critical Nonlinearity on $R^{N}$. Journal of Mathematical Analysis Application, 434, 1826-1851. https://doi.org/10.1016/j.jmaa.2015.10.013

[3] Yang, Z.J., Ding, P.Y. and Li, L. (2016) Longtime Dynamics of the Kirchhoff Equations with Fractional Damping and Supercritical Nonlinearity. Journal of Mathematical Analysis Application, 442, 485-510. https://doi.org/10.1016/j.jmaa.2016.04.079

[4] Igor, C. (2012) Longtime Dynamics of Kirchhoff Wave Models with Strong Nonlinear Damping. Journal of Differential Equations, 252, 1229-1262. https://doi.org/10.1016/j.jde.2011.08.022

[5] Varga, K. and Sergey, Z. (2009) Finite-Dimensional Attractors for the Quasi-Linear StronglyDamped Wave Equation. Journal of Differential Equations, 247, 1120-1155. https://doi.org/10.1016/j.jde.2009.04.010

[6] Lin, X.L. and Li, F.S. (2013) Global Existence and Decay Estimates for Nonlinear KirchhoffType Equation with Boundary Dissipation. Differential Equations and Applications, 5, 297 317. https://doi.org/10.7153/dea-05-18

[7] Li, F.C. (2004) Global Existence and Blow-Up of Solutions for a Higher-Order KirchhoffType Equation with Nonlinear Dissipation. Applied Mathematics Letters, 17, 1409-1414. https://doi.org/10.1016/j.am1.2003.07.014

[8] Salim, A.M. and Belkacem, S.H. (2007) A Blow-Up Result for a Higher-Order Nonlinear Kirchhoff-Type Hyperbolic Equation. Applied Mathematics Letters, 20, 866-871. https://doi.org/10.1016/j.aml.2006.08.018

[9] Gao, Q.Y., Li, F. and Wang, Y.G. (2010) Blow-Up of the Solution for Higher-Order Kirchhoff-Type Equations with Nonlinear Dissipation. Central European Journal of Mathematics, 9, 686-698. https://doi.org/10.2478/s11533-010-0096-2

[10] Lou, R.J., Lv, P.H. and Lin, G.G. (2016) Global Attractors for a class of Generalized Nonlinear Kirchhoff-Sine-Gordon Equation. International Journal of Modern Nonlinear Theory and Application, 5, 73-81. https://doi.org/10.4236/ijmnta.2016.51008

[11] Lin, G.G. (2011) Nonlinear Evolution Equation. Yunnan University Press, Kunming. 
Submit or recommend next manuscript to SCIRP and we will provide best service for you:

Accepting pre-submission inquiries through Email, Facebook, LinkedIn, Twitter, etc. A wide selection of journals (inclusive of 9 subjects, more than 200 journals)

Providing 24-hour high-quality service

User-friendly online submission system

Fair and swift peer-review system

Efficient typesetting and proofreading procedure

Display of the result of downloads and visits, as well as the number of cited articles

Maximum dissemination of your research work

Submit your manuscript at: http://papersubmission.scirp.org/

Or contact ijmnta@scirp.org 